\title{
Renal replacement treatment for diabetic patients in Newcastle upon Tyne and the Northern region, 1964-88
}

\author{
C Catalano, T H J Goodship, J S Tapson, M K Venning, R M R Taylor, G Proud, W M G Tunbridge, \\ $\mathrm{R}$ W Elliot, M K Ward, K G M M Alberti, R Wilkinson
}

\begin{abstract}
Objectives-To review the experience of renal replacement treatment in diabetic patients treated in Newcastle upon Tyne and the Northern region from 1964 to 1988 , and to compare the morbidity and mortality of diabetic patients treated with dialysis or transplantation with those of matched controls of non-diabetic patients.

Design-Retrospective study of clinical case notes.

Setting-Renal units of the Northern region, particularly that in Newcastle upon Tyne.

Patients-All 65 diabetic patients treated by renal replacement treatment in Newcastle upon Tyne from 1964 to $1987 ; 42$ diabetic patients were matched with 42 non-diabetic patients according to age, sex, year of starting treatment, and type of treatment (dialysis or transplantation).
\end{abstract}

Main outcome measures-Sex, age, renal biopsy findings, blood pressure, history of diabetic treatment, and plasma creatinine concentration at the start of renal replacement treatment. History of renal replacement treatments, suitability for transplantation, history of transplantation, cumulative survival, and cause of death during follow up. Survival of technique, cumulative survival of the first peritoneal catheter and history of peritonitis in patients treated with continuous ambulatory peritoneal dialysis; source of graft, histocompatibility antigens, duration of associated stay in hospital, and graft survival in patients receiving renal or pancreatic transplant.

Results-1259 Patients with chronic renal failure were accepted for renal replacement treatment in Newcastle upon Tyne, of whom 65(5\%) had diabetes. The first was accepted in 1974, and between 1974 and 1980 another 15 were treated (mean age 42 years; $4 \%$ of new patients). From 1981 to 1987,49 diabetic patients (mean age $44 ; 9 \%$ of new patients) were treated. Fifty patients $(\mathbf{7 7 \%})$ had insulin dependent diabetes and the remaining $15(23 \%)$ non-insulin dependent diabetes. On average, the patients were aged 25 (range 5-57) when diabetes was first diagnosed and 44 (range 24-70) at the start of renal replacement treatment. The mean age at the start of treatment was $\mathbf{4 0}$ for patients with insulin dependent diabetes and $\mathbf{5 8}$ for patients with non-insulin dependent diabetes. Transplantation was performed in 33 of the diabetic patients, whose mean age was lower than that of those who did not receive a transplant ( $41 v 48$ respectively, $\mathrm{p}<0.05$ ). Comparison between the 42 diabetic patients and matched controls showed that the overall survival at five years was $46 \%$ and $77 \%$ respectively. The three year survival of the diabetic patients who did not receive a transplant was poor ( $41 \% v 79 \%$ respectively). Of patients transplanted, survival at five years was $73 \%$ in the diabetic patients and $90 \%$ in the controls. However, there was no significant difference in the five year graft survival ( $64 \% v 46 \%$ respectively).

Conclusions-Diabetes adversely affects morbidity and mortality in patients having renal replacement treatment, but renal transplantation seems to be the best option for treating diabetic patients with end stage renal failure.

\section{Introduction}

End stage renal failure is an important cause of death in diabetic patients, especially in those in whom diabetes develops at a young age. In the United Kingdom in 1979 it was the cause of death in $15 \%$ of those aged under 50 and a quarter of those in whom it was diagnosed before the age of $31 .^{1}$ About a fifth of insulin dependent diabetic patients will develop end stage renal failure within 25 years after diagnosis, ${ }^{2}$ although this incidence may be falling.

When renal replacement treatment first became available diabetic patients were often not considered for treatment because of limited facilities. In the past 10 years, however, increasing numbers of diabetic patients have been accepted for treatment and in some countries diabetic nephropathy is now the most common diagnosis among patients newly accepted for dialysis. ${ }^{34}$

Many reports have described experiences with renal replacement treatment in diabetic patients, ${ }^{3-15}$ several of which compared findings with a control group, ${ }^{10121}$ but to our knowledge in none has a comparison been made with a matched control group of non-diabetic patients treated in the same unit. In this study we reviewed the experience of renal replacement treatment in diabetic patients treated in Newcastle upon Tyne and the Northern region. We also compared the morbidity and mortality of a group of diabetic patients treated with dialysis or transplantation, or both, with those of a matched control group of non-diabetic patients.

\section{Methods}

\section{ACCEPTANCE RATES IN THE NORTHERN REGION}

In the Northern region renal replacement treatment is available in renal units in Newcastle upon Tyne, Sunderland, and Middlesbrough, which opened in 1964, 1968, and 1969 respectively. Newcastle upon Tyne is also the regional centre for kidney and pancreatic transplantation. The acceptance rate by year per million population was calculated from the total number of diabetic and non-diabetic patients accepted for renal replacement treatment in the three units. Figures for the population served by the Northern Regional Health Authority from 1964 were supplied by the statistics section of the health authority. 
RENAL REPLACEMENT TREATMENT OF DIABETIC PATIENTS IN NEWCASTLE UPON TYNE

The clinical notes of all diabetic patients accepted for renal replacement treatment in Newcastle upon Tyne from 1964 to 1987 were reviewed and the following data obtained: sex, age, findings on renal biopsy, blood pressure and plasma creatinine concentration at the start of treatment, history of treatment, suitability for transplantation, history of admissions, cumulative survival, and cause of death. Diabetes was classified as either insulin dependent or non-insulin dependent, according to the treatment instituted at the time of diagnosis and at one year. Patients treated with insulin within the first year of treatment were considered to have insulin-dependent diabetes whereas those who were treated for at least one year with oral hypoglycaemic agents or diet, or both, were considered to have non-insulin-dependent diabetes.

In all patients treated by continuous ambulatory peritoneal dialysis the following were also recorded: survival of the technique, cumulative survival of the first catheter, survival of the catheter to the first episode of peritonitis, the number of episodes of peritonitis, and the cause (fungal or bacterial) in each. Failure of the technique was considered to have occurred if continuous ambulatory peritoneal dialysis was permanently discontinued for one of the following reasons: peritonitis, loss of ultrafiltration, persistent nausea and vomiting, and patient non-compliance. Loss of the first catheter was considered to have occurred if its removal was necessary because of persistent peritonitis and complications related to the catheter itself. Removal of the catheter after successful kidney transplantation was considered to be a censored observation.

In all patients having renal and pancreatic transplantation the following were recorded: source of the graft (live or cadaveric donor), histocompatibility antigens of patient and donor, duration of stay in hospital related to transplantation, and survival of the kidney and the pancreas. The function of pancreatic grafts was defined as a reduction, of at least half, in insulin requirements and evidence of endogenous insulin secretion from $\mathrm{C}$ peptide measurements.

COMPARISON WITH MATCHED CONTROL GROUP OF NON-DIABETIC PATIENTS

An attempt was made to match all the diabetic patients with non-diabetic patients according to the following criteria: sex, age at onset of treatment, year in which treatment started (up to 1987), and the type of treatment (dialysis or transplantation, or both). Patients with myeloma, amyloidosis, or obstructive

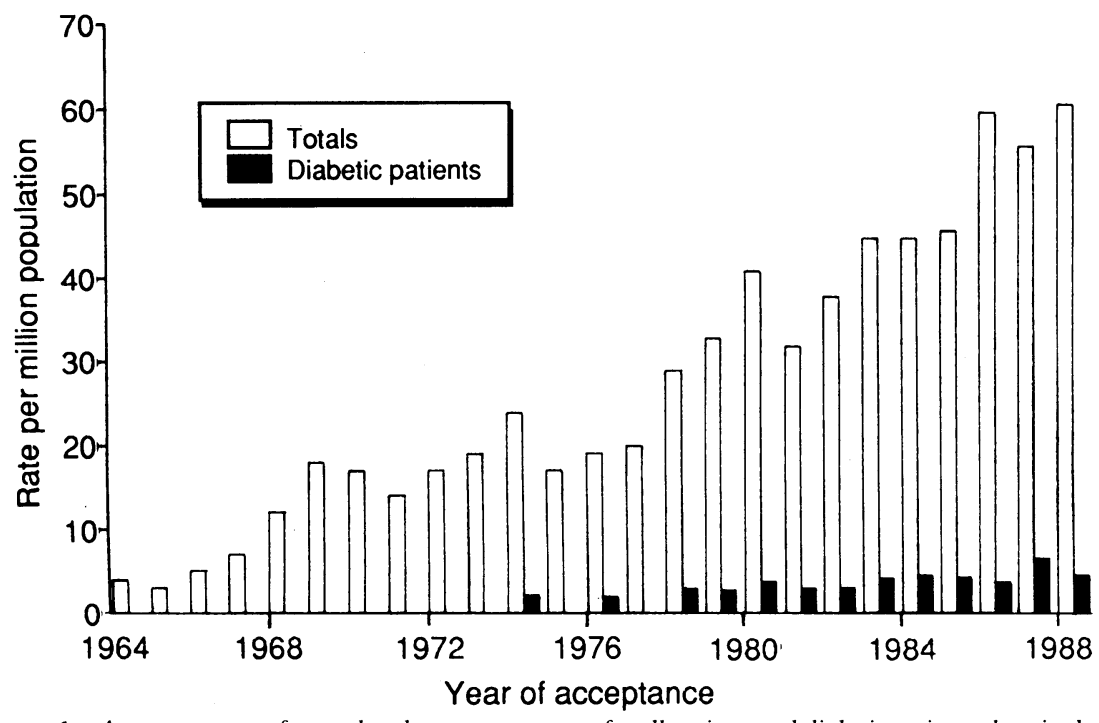

FIG 1 -Acceptance rates for renal replacement treatment for all patients and diabetic patients alone in the Northern region, $1964-88$ uropathy secondary to malignancy were not considered as controls. It was possible to match 42 diabetic patients. The morbidity and mortality in the two groups were compared.

\section{STATISTICAL ANALYSIS}

The two groups were compared with Student's unpaired $t$ test and by $\chi^{2}$ analysis. For analysis of survival we used the method of Kaplan and Meier, ${ }^{16}$ and survival was compared with the log rank test. ${ }^{17}$ Analysis of survival continued up to the end of 1988. In analysing graft survival, death in patients with a functioning transplant were considered as censored observations. All values are expressed as means (SEM) unless otherwise stated.

\section{Results}

Acceptance rates in the Northern region-From 1964 to 1987, 1950 patients were accepted for renal replacement treatment in the Northern region and 105 of these $(5 \cdot 4 \%)$ had diabetes. The first diabetic patient started treatment in 1974. Figure 1 shows the change in acceptance rate in this period. The total acceptance rate rose steadily, though the increase in the acceptance rate among diabetic patients was less pronounced.

Treatment for diabetic patients in Newcastle upon Tyne-From 1964 to 1987, 1259 patients started renal replacement treatment, $65(5 \cdot 2 \%)$ of whom had diabetes. Only one of the diabetic patients was not of European origin. The mean duration of observation was 35 (range 2-108) months. The patients' mean age was 25 (2) at diagnosis and 44 (2) at the onset of renal treatment. Fifty patients $(77 \%)$ had insulin dependent and $15(23 \%)$ non-insulin dependent diabetes, and their mean age at the start of renal replacement treatment was 40 (2) and 58 (3) respectively. The mean duration of treatment (with diet or hypoglycaemic drugs, or both) in the patients with non-insulin dependent diabetes was 11 (2) years. Only one patient with non-insulin dependent diabetes was treated with oral hypoglycaemic drugs for one year. Renal biopsy was performed in $12(18 \%)$ patients, in whom some doubt existed about pathogenesis of the disease; diabetic nephropathy was confirmed in nine and glomerulonephritis was found in the three others. Fifty three $(82 \%)$ patients were treated initially with peritoneal dialysis, $10(15 \%)$ with haemodialysis and two (3\%) received a transplant before the onset of dialysis.

Renal and pancreatic transplantation-Fifty two patients $(80 \%)$ were accepted for renal transplantation and thereafter $33(51 \%)$ received a transplant. The mean age of the patients who received a transplant was significantly lower than that of those did not $(41$ (2) $v 48$ $(2), p<0.05)$. Of these 33 patients, 22 had been previously treated with peritoneal dialysis, nine with haemodialysis, and two had received transplants before dialysis. Six patients had a combined pancreatic and renal transplant. The survival of the pancreatic graft in these patients was 54 months (censored observation), 11 months (censored observation), and 2, 0, 0, and 0 months respectively. Ten of the patients had a biopsy of the transplanted kidney for "late" progressive deterioration in renal function, and in only one was severe diabetic nephropathy found; the others showed various degrees of chronic rejection. The mean duration of stay in hospital related to transplantation was $\mathbf{5 0}$ days for combined pancreatic and renal transplants and 28 days for a kidney transplant alone.

Comparison with matched control group of non-diabetic patients - Forty two of the diabetic patients could be matched in the controls. The mean age at the onset of renal replacement treatment was the same in both groups (diabetic patients 43 (2) $v$ controls 43 (2), and the mean duration of observation was 42 (range 2-103) 
months in the diabetic patients and 58 (range 2-177) months in the controls. Plasma creatinine concentration was slightly lower at the start of renal replacement treatment in the diabetic patients (947 (47) $v 1053$ (39) $\mu \mathrm{mol} / 1$, not significant). Systolic and diastolic blood pressures were similar at the onset of the treatment (diabetic patients 168/89 $v$ controls $163 / 97 \mathrm{~mm} \mathrm{Hg}$ ) but more diabetic patients were being treated with antihypertensive drugs ( $32 v 27$, not significant).

Transplantation - In each group 34 patients $(81 \%)$ were considered suitable for transplantation, and 23 patients received a renal transplant. The mean age of the patients who received transplants was significantly lower than that of those who did not (diabetic patients 36 (2) v 52 (2); controls 37 (2) $v 51$ (2); both $\mathrm{p}<0 \cdot 01$ ). Two diabetic patients received a kidney transplant from a living relative. The number of matches at each locus of the major histocompatibility complex was similar in the two groups (diabetics A-16, B-14, Dr-11; controls A-17, B-22, Dr-5), as was the number of rejection episodes (diabetics 24; controls 23 ).

Survival of the transplanted kidney in the patients receiving a combined kidney and pancreas transplant was no different from that in their matched controls (diabetics 29 months $v$ controls 24 months). Only one of the patients receiving a pancreatic transplant died during the observation period.

Admission-Both the rate of admission and duration of stay in hospital were significantly higher in the diabetic patients (diabetic patients $2 \cdot 13 v$ controls $1 \cdot 37$ admissions year, $\mathrm{p}<0.001$; diabetic patients $29.1 \mathrm{v}$ controls $17 \cdot 1$ days year, $\mathrm{p}<0.001$ respectively). Admissions were significantly related to either peripheral vascular disease or urinary infections in the diabetic group compared with controls $(17 \cdot 3 \% v 1 \cdot 5 \%$, $\mathrm{p}<0.01$, table I). Moreover, the diabetic patients were more frequently admitted for infections, nausea, vomiting, and assessment of poor glycaemic control. Diabetic patients receiving transplants were admitted slightly more frequently for infection, but this trend did not reach significance.

TABLE I-Reasons for admission in 42 diabetic patients and matched controls. Figures are numbers (percentages)

\begin{tabular}{lcc}
\hline & Diabetic patients & Controls \\
\hline Problem related to graft & $43(18 \cdot 6)$ & $41(20 \cdot 5)$ \\
Peritonitis & $37(16 \cdot 0)$ & $26(13 \cdot 0)$ \\
Amputations or gangrene & $29(12 \cdot 6)$ & $2(1 \cdot 0)^{\star}$ \\
Problems of access & $27(11 \cdot 7)$ & $30(15 \cdot 0)$ \\
Urinary tract infection & $11(4 \cdot 8)$ & $1(0 \cdot 5)^{\star}$ \\
Infections or fever & $16(6 \cdot 9)$ & $8(4 \cdot 0)$ \\
Diabetic control & $12(5 \cdot 2)$ & $2(1 \cdot 0)$ \\
Nausea and vomiting & $8(3 \cdot 5)$ & \\
Visual problems & $3(1 \cdot 3)$ & \\
Hypertension & $2(0 \cdot 9)$ & $3(1 \cdot 5)$ \\
Fluid overload & $1(0 \cdot 4)$ & $87(43 \cdot 5)$ \\
Others & $42(18 \cdot 0)$ & 200 \\
\hline Total & 231 & \\
\hline
\end{tabular}

${ }^{\star} \mathrm{p}<0.01$

Continuous ambulatory peritoneal dialysis-Sixteen patients in each group were treated initially by continuous ambulatory peritoneal dialysis. Survival of the diabetic patients was lower than that of the controls, although the difference was not significantly different (fig 2). Survival of the technique was similar in the two groups (fig 2), and there was no difference in either the time to the first episode of peritonitis (diabetic patients 28 (9) $v$ controls 28 (11) weeks) or the survival of the first peritoneal catheter (fig 2). Although survival of the catheter to the first episode of peritonitis was similar in the two groups, the overall rates of bacterial and fungal peritonitis were significantly higher in the diabetic patients (bacterial peritonitis, one episode $/ 6 \cdot 1$ patient months $v$ one episode $/ 12.4$ patient months, $\mathrm{p}<0.001$; fungal peritonitis, one episode/ 74 patient months $v$ one episode/564 patient months, $\mathrm{p}<0.05)$. Five
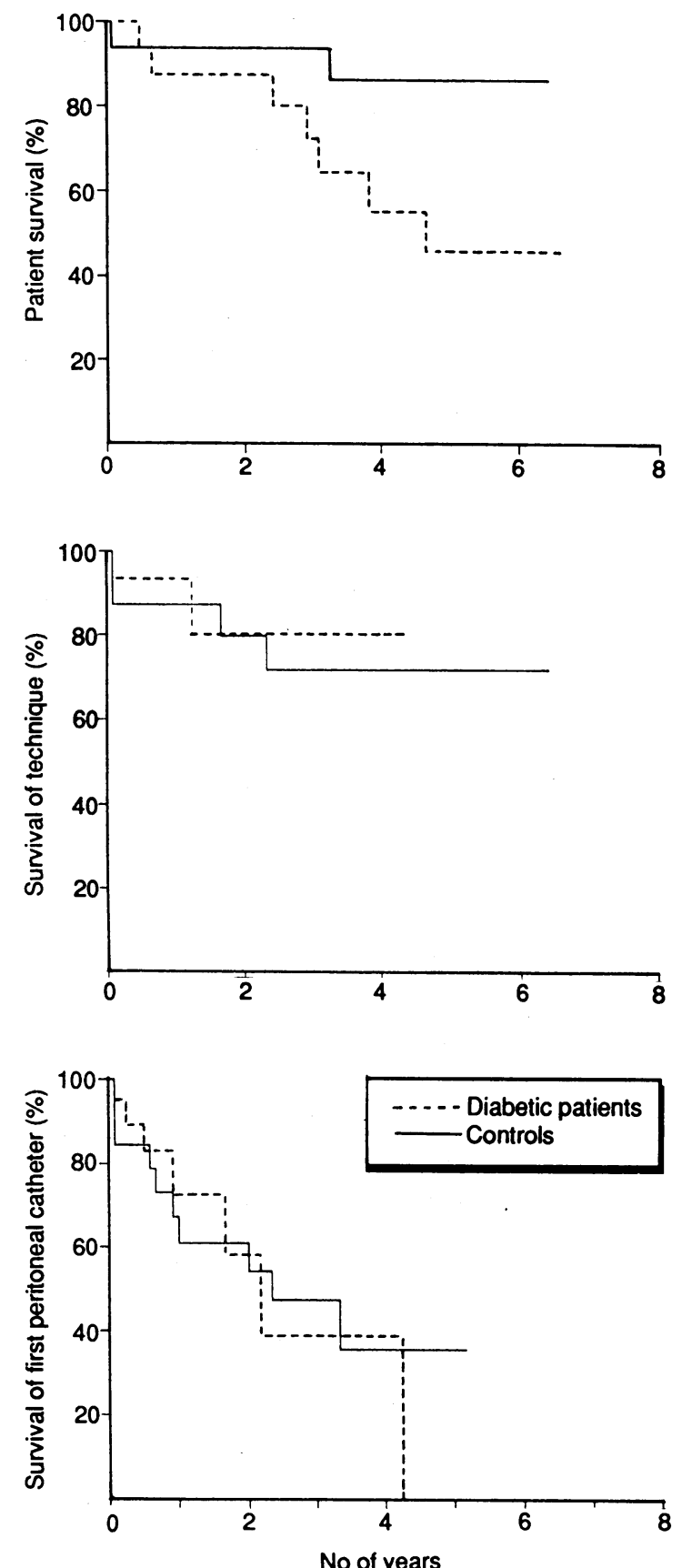

FIG 2-Survival analyses in diabetic patients and controls treated with continuous ambulatory peritoneal dialysis. Top: overall patient survival; middle: survival of technique; bottom: survival of first peritoneal catheter; log rank test NS

diabetic patients and two controls each had one episode of fungal peritonitis. The incidence of peritonitis was not different in diabetic patients with and without severe bilateral visual impairment.

Diabetic complications-Before starting renal replacement treatment $38(90 \%)$ of the diabetic patients had diabetic retinopathy and $17(40 \%)$ had severe bilateral reduction of their sight. Only one patient out of $25(4 \%)$ became blind after starting the treatment; 14 $(33 \%)$ of the diabetic patients had one or more amputations, and five (12\%) presented with a cerebrovascular accident, which in all was eventually fatal. In comparison, only one patient in the control group had an amputation (of a finger), and two had non-fatal cerebrovascular accidents.

Survival-Overall cumulative survival of the diabetic patients was significantly worse than that in the controls ( $\log$ rank test, $\mathbf{p}<0.01$ ) (fig 3 ). Five year survival of those patients receiving a transplant was $73 \%$ in the diabetic patients and $90 \%$ in the controls (log rank test, $\mathrm{p}<0.05$ ) (fig 4) and zero and $60 \%$ 
respectively in those patients not receiving a transplant ( $\log$ rank test, $p<0.001$ ) (fig 5). Cumulative survival of the transplanted kidney (fig 6) was not significantly different in the two groups (one year survival in diabetic patients $73 \% v$ controls $70 \%$; five year survival in diabetic patients $64 \% v$ controls $46 \%$ ).

Cause of death-Cerebrovascular accidents were a significant cause of death in only the diabetic patients (table II). Otherwise, the cause of death was similar in the two groups: two patients in the diabetic group and one in the control group discontinued dialysis, and one patient in the control group committed suicide.

\section{Discussion}

From 1964 to $1987,5 \cdot 4 \%$ of all patients accepted for renal replacement treatment in the Northern region were diabetic patients. The first of these was accepted

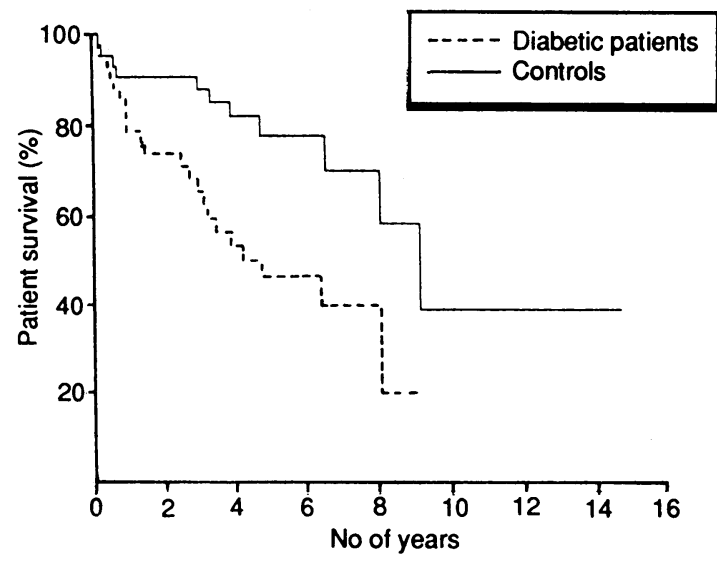

FIG 3-Overall survival of diabetic patients and controls having all forms of renal replacement treatment; log rank test $p<0.01$

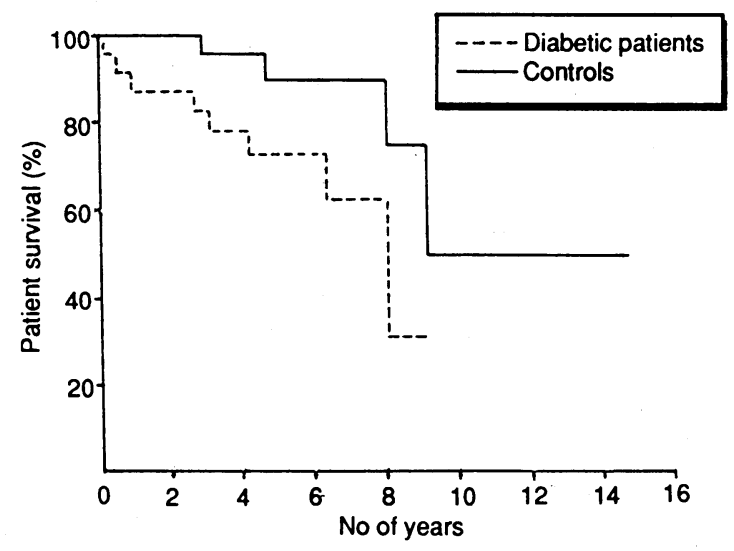

FIG 4-Survival of diabetic patients and controls receiving renal transplant; log rank test $p<0.05$

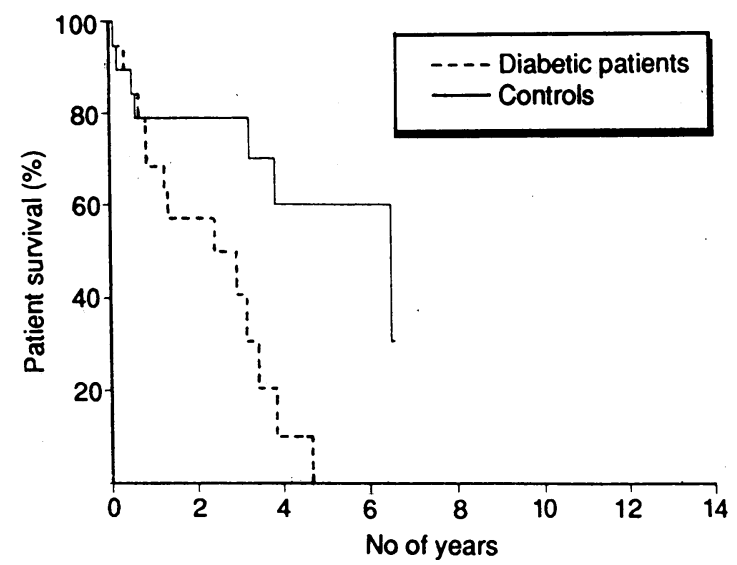

FIG 5-Survival of diabetic patients and controls not receiving renal transplant; log rank test $p<0.001$

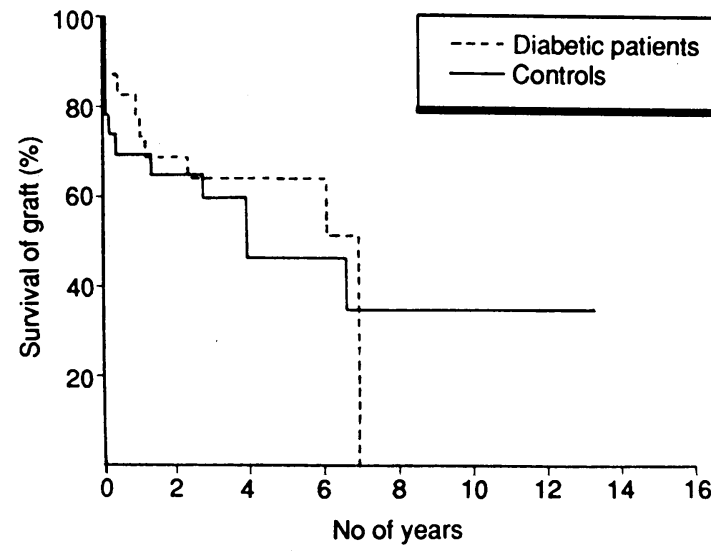

FIG 6-Survival of graft in diabetic patients and controls receiving renal transplant; log rank test $N S$

TABLE II-Causes of death in 42 diabetic patients and matched controls. Figures are numbers (percentages)

\begin{tabular}{lcc}
\hline Cause of death & Diabetic patients & Controls \\
\hline Vascular & $5(23)$ & \\
Cardiac & $7(32)$ & $4(33)$ \\
Infection & $5(22)$ & $2(17)$ \\
Social & $2(9)$ & $2(17)$ \\
Other & $3(14)$ & $4(33)$ \\
\hline Total & 22 & 12 \\
\hline
\end{tabular}

* Suicide and discontinuation of dialysis.

in the Newcastle unit in 1974, 10 years after the start of the dialysis programme. Thereafter, the acceptance rate for diabetic patients increased steadily and in 1985 was 4.5 patients per million population. This figure is similar to those reported by the European Dialysis and Transplant Association registry for the United Kingdom and Europe in 1985 (4.3 and 3.6 per million population respectively). ${ }^{3}$ The corresponding acceptance rate for the United States for 1985 reported by Medicare is, however, remarkable in comparison (32 per million) $)^{18}$ and approaches the total acceptance rate for the United Kingdom in 1985 (38 per million). ${ }^{3}$

In the United States about half of diabetic patients having haemodialysis have non-insulin dependent diabetes $^{19}$ whereas most of our patients had insulin dependent diabetes, and this is reflected by the younger age of our patients $(44 v 60)$.

Renal biopsy is performed in our diabetic patients only when the primary renal diagnosis is uncertain, when patients present without proliferative retinopathy, with diabetes of a short duration, and with an unexplainable, unexpected deterioration in renal function. Twelve patients had a biopsy and three $(25 \%)$ were found to have non-diabetic renal disease. This proportion is similar to that in selected populations of diabetics with renal impairment. ${ }^{20-22}$ In only one of 10 transplanted kidneys was recurrent diabetic glomerulosclerosis found on biopsy. This low incidence may be related to the fairly short period of observation. Goetz et al reported a higher incidence of recurrence in diabetic patients 10 years after transplantation. ${ }^{6}$

Over $80 \%$ of our diabetic patients were treated initially with peritoneal dialysis $(80 \%$ with continuous ambulatory peritoneal dialysis). This rate represents the popularity of peritoneal dialysis in Newcastle upon Tyne $(60 \%$ of dialysis patients in 1988 received continuous ambulatory peritoneal dialysis) and is higher than that for the rest of the United Kingdom and Europe $(21 \%$ and $7 \%$ respectively of all dialysis patients, $43 \%$ and $17 \%$ of diabetic patients (European Dialysis and Transplant Association registry, personal communication). In Minnesota only $5 \%$ of diabetic patients are treated by peritoneal dialysis. ${ }^{14}$ We believe that the use of this type of dialysis in diabetic patients is associated with many advantages. It is easy to perform, 
fairly inexpensive, and allows independence of the patients; blood pressure, fluid balance, and bone disease are well controlled. Moreover, in diabetic patients giving intraperitoneal insulin allows good glycaemic control. ${ }^{23}$

In our experience the survival of the diabetic patients receiving continuous ambulatory peritoneal dialysis was worse than that of the controls, although this trend failed to reach significance. Survival of the technique survival and the survival of the first peritoneal catheter was, however, similar in both groups. The four year survival of diabetic patients treated by continuous ambulatory peritoneal dialysis was similar to that reported by the European Dialysis and Transplant Association registry ${ }^{12}$ for diabetic patients aged between 15 and 44 years (Newcastle 55\% v Europe $58 \%)$ and better than that reported by Berisa et al $(32 \%)^{10}$ despite a similar age group; in neither of these reports were matched controls used. The crude survival of our diabetic patients was similar to $^{24}$ or better than ${ }^{25}$ other uncontrolled studies. Comparing survival with continuous ambulatory peritoneal dialysis and with haemodialysis was not possible because of the small numbers of diabetic patients treated by haemodialysis (continuous ambulatory peritoneal dialysis 53 , haemodialysis 10 ).

The incidence of bacterial and fungal peritonitis was higher in our diabetic patients than in the controls. Survival of technique and time to the first episode of peritonitis were, however, similar in the two groups. Kraus and Spector reported no difference in the rate of bacterial peritonitis in their diabetic and non-diabetic patients, ${ }^{26}$ but the two groups were not matched. Legrain et $\mathrm{l}^{8}$ and Passlick and Grabensee ${ }^{25}$ reported, in uncontrolled studies, a lower rate of bacterial peritonitis in diabetic patients (one episode/13.2 patient months and one episode/ 18 patient months respectively).

Most $(80 \%)$ of our patients were considered suitable for transplantation, and $51 \%$ received a transplant. The little information available about either the acceptance rate or transplantation rate for diabetic patients in other centres shows a wide variation in practice. The Minnesota group reported that over 16 years 56 out of a total of 369 diabetic patients $(15 \%)$ received a transplant ${ }^{14}$ whereas at King's College Hospital $77 \%$ of diabetic patients did so. ${ }^{15}$ Transplantation is, however, generally accepted as the best treatment for diabetic patients with end stage renal failure with respect to survival and rehabilitation. ${ }^{27}$

In this study the rate of admission and duration of stay in hospital were higher in the diabetic group and greater than those reported by the Minnesota group ${ }^{14}$ $(2.1 v 1.4$ admissions/year, $29 v 12$ days/year in hospital respectively) whereas Legrain et al reported a longer duration of stay in hospital in diabetic patients treated with continuous ambulatory peritoneal dialysis (42 days/year) or haemodialysis (46 days/year). ${ }^{8}$

Admissions in diabetic patients receiving dialysis are commonly related to complications of peripheral vascular disease and to infections. ${ }^{11} 14$ We are unaware, however, that a high incidence of urinary tract infections has been found previously in such patients. In 1972 Ghavamian et al expressed concern at the rapid deterioration in visual acuity found in diabetic patients receiving haemodialysis. ${ }^{28}$ That this is now less commonly reported ${ }^{11}$ may reflect the higher rate of transplantation, increased use of peritoneal dialysis, and meticulous attention to ophthalmic care. In our experience only one patient showed evidence of deterioration in visual acuity after the onset of renal replacement treatment. The incidence of amputations and cerebrovascular accidents in our diabetic patients was high. This has been commonly reported even in patients with functioning transplants ${ }^{891115}$ and probably reflects the inexorable progression of arterial disease.

The overall survival at five years of our control population was similar to that reported by the European Dialysis and Transplant Association registry for patients aged between 35 and 45 (77\% $v 75 \%$ respectively) whereas the survival of diabetic patients was better $\left(46 \% v 37 \%\right.$ respectively). ${ }^{12}$ This may be explained by the high percentage of patients receiving transplants in Newcastle upon Tyne. The overall five year survival reported by the Minnesota group was worse $(25 \% v 46 \%)$, but the mean age of their patients was higher $(51 v 43)$ and only $15 \%$ had received a transplant. ${ }^{14}$ The five year survival of diabetic patients who received a transplant $(73 \%)$ is better than that in other reported series $\left(47 \%{ }^{15} ; 35 \%{ }^{12} ; 40 \%{ }^{13}\right)$ and suggests that renal transplantation is the best mode of treatment for diabetic patients. The five year survival of diabetic patients who did not receive a transplant was zero, which is worse than the figures of the European Dialysis and Transplant Association registry (haemodialysis $30 \%$; continuous ambulatory peritoneal dialysis $\left.58 \%{ }^{12}\right)$. The poor results in this group may be explained by selection of the younger, fitter patients for transplantation. Survival of the graft in the diabetic patients and controls was similar, and the three year graft survival in the diabetic patients was better than that reported by the European Dialysis and Transplant Association registry $\left(64 \% v 45 \%{ }^{12}\right)$. Our experience of pancreatic transplantation has been small, and the results of graft survival and duration of stay in hospital were disappointing. Recent advances in techniques for pancreatic transplantation, however, have resulted in improved figures. Moreover, loss of the pancreatic transplant did not seem to jeopardise the patient's life nor affect the survival of the transplanted kidney.

Twenty three per cent of the deaths in our diabetic patients were the result of cerebrovascular accidents whereas no deaths from this cause occurred in the control group. Cerebrovascular accidents are both more common and more often fatal in diabetic patients with and without diabetic nephropathy. ${ }^{11} 29$

In summary, our experience of renal replacement treatment in diabetic patients is similar to that of others. Diabetes strongly influences both morbidity and mortality; however, the six year survival of diabetic patients receiving a kidney transplant is over $70 \%$.

CC was supported by Consiglio Nazionale delle Ricerche, Italy, and THJG by the Northern Counties Kidney Research Fund. We acknowledge the help of Mrs Jo Little in collecting data and are grateful to Mrs S Dikes for data from the European Dialysis and Transplant Association registry, to Dr M McHugh, Sunderland Royal Infirmary, and Dr R Cove-Smith, South Cleveland Hospital, for information about the patients treated in their units.

1 Moloney A, Tunbridge WMG, Ireland JT, Watkins PJ. Mortality from diabetic nephropathy in the United Kingdom. Diabetologia 1983;25:26-30.

2 Andersen AR, Christiansen JS, Andersen JK, Kreiner S, Deckert T. Diabetic nephropathy in type 1 (insulin-dependent) diabetes: an epidemiological study. Diabetologia 1983;25:496-501.

3 Brunner FP, Brynger H, Challah S, et al. Renal replacement therapy in patients with diabetic nephropathy, 1980-1985. Report from the European patients with diabetic nephropathy, 1980-1985. Report from the European 1988;3:585-95.

4 Eggers PW. Effect of transplantation on the medicare end-stage renal disease program. N Engl f Med 1988;318:223-9.

5 Mailloux LU, Bellusi AG, Mossey RT, et al. Predictors of survival in patients undergoing dialysis. Am $\mathcal{F}$ Med 1988;84:855-61.

6 Goetz FC, Elick B, Fryd D, Sutherland DER. Renal transplantation in diabetes. In: Watkins PJ, ed. Clinics in endocrinology and metabolism. Long term complications of diabetes. London: W B Saunders, 1986:807-21.

7 Rosansky SJ, Eggers PW. Trends in the US end-stage renal disease population: 1973-1983. Am $\mathcal{F}$ Kidney Dis 1987;9:91-7.

8 Legrain M, Rottembourg J, Bentchikou A, et al. Dialysis treatment of insulin dependent diabetic patients: ten years' experience. Clin Nephrol 1984;21:7281 .

9 Khanna R, Oreopoulos DG. Dialysis: continuous ambulatory peritonea dialysis and haemodialysis. In: Watkins PJ, ed. Clinics in endocrinology and metabolism. Long term complications of diabetes. London: W B Saunders, 1986:823-6. 
10 Berisa F, McGonigle R, Beaman M, Adu D, Micheal J. The treatment of diabetic renal failure by continuous ambulatory peritoneal dialysis. Diabetic Med 1989;6:67-70

11 Kjellstrand CM, Whitley K, Comty CM, Shapiro FL. Dialysis in patients with diabetes mellitus. Diabetic Nephropathy 1983;2:5-17.

12 Brunner FP, Broyer M, Brynger H, et al. Survival on renal replacement therapy: data from the EDTA registry. Nephrol Dial Transplant 1988;2:109. 22.

13 Larsson O, Attman P, Aurell M, Frisk B, Brynger $\mathrm{H}$. Survival in end-stag diabetic renal disease. A prospective study of 100 kidney transplant patients. Acta Med Scand 1984;216:393-402.

14 Matson M, Kjellstrand CM. Long-term follow-up of 369 diabetic patients undergoing dialysis. Arch Intern Med 1988;148:600-4.

15 Grenfell A, Bewick M, Parsons V, Snowden S, Taube D, Watkins PJ. Noninsulin-dependent diabetes and renal replacement therapy. Diabetic Med 1988;5:172-6

16 Kaplan EL, Meier P. Non-parametric estimation from incomplete observations. Fournal of the American Statistical Association 1958;53:457-81.

17 Peto R, Pike MC, Armitage P, et al. Design and analysis of randomized clinical trials requiring prolonged observation of each patient. II. Analysis and examples. Br f Cancer 1977;35: 1-39.

18 Teutsch S, Newman J, Eggers $P$. The problem of diabetic renal failure in the United States: an overview. Am f Kidney Dis 1989;13:11-3.

19 Herman W, Hawthorne V, Hamman R, et al. Preventing the kidney disease of diabetes mellitus: public health perspectives. Consensus statement. $A m \mathcal{F}$ Kidnev Dis 1989:13:2-10.

20 Lynn KL, Frendin TJ, Walker RJ, Bailey RR, Swainson CP. Renal disease in diabetics - which patients have diabetic neophropathy and what is their outcome? Aust NZ F Med 1988;18:764-7.

21 Chihara J, Takebayashi S, Taguchi T, Yokoyama K, Harada T, Naito S Glomerulonephritis in diabetic patients and its effect on their prognosis. Nephron 1986;43:45-9.

22 Kasinath BS, Mujais SK, Spargo BH, Katz AI. Nondiabetic renal disease in patients with diabetes mellitus. Am $\mathcal{F}$. Med 1983;75:613-7.

23 Heaton A, Rodger RSC, Sellars L, et al. Continuous ambulatory peritoneal dialysis after the honeymoon: review of experience in Newcastle 1979-84 Br.Med f 1986;293:938-41.

24 Amair $\mathrm{P}$, Khanna R, Leibel B, et al. Continuous ambulatory peritoneal dialysis in diabetics with end-stage renal disease. $N$ Englf $M$ Med 1982;306:625-30.

25 Passlick J, Grabensee B. CAPD and transplantation in diabetics. Clin Nephrol 1988;30(suppl 1):S18-23.

26 Kraus S, Spector DA. Characteristics and sequelae of peritonitis in diabetics and nondiabetics receiving chronic intermittent peritoneal dialysis. Medicine 1983;62:52-7.

27 Manis T, Friedman EA. Current thinking on the management of the uremic diabetic. Semin Nephrol 1986;6:183-5.

28 Ghavamian M, Gutch CF, Kopp KF, Kolff WJ. The sad truth abou haemodialysis in diabetic nephropathy. FAMA 1972;222:1386-9.

29 Pyorala K, Laakso M. Macrovascular disease in diabetes mellitus. In: Mann JI, Pyorala K, Teuscher A, eds. Diabetes in epidemiological perspective. London: Churchill Livingstone, 1983:183-247.

Accepted 18 fune 1990)

\title{
Outcome of renal replacement treatment in patients with diabetes mellitus
}

\author{
Margaret A McMillan, J Douglas Briggs, Brian J R Junor
}

Abstract

Objective-To compare the outcome of renal replacement treatment in patients with diabetes mellitus and in non-diabetic patients with end stage renal failure.

Design-Retrospective comparison of cases and matched controls.

Setting - Renal unit, Western Infirmary, Glasgow, providing both dialysis and renal transplantation.

Patients-82 Diabetic patients starting renal replacement treatment between 1979 and 1988, compared with 82 matched non-diabetic controls with renal failure and 39 different matched controls undergoing renal transplantation.

Main outcome measures-Patient characteristics, history of smoking, prevalence of left ventricular hypertrophy and myocardial ischaemia at start of renal replacement treatment; survival of patients with renal replacement treatment and of patients and allografts with renal transplantation.

Results-The overall survival of the diabetic patients during the treatment was $83 \%, 59 \%$, and $50 \%$ at one, three, and five years. Survival was significantly poorer in the diabetic patients than the controls $(p<0 \cdot 001)$. Particularly adverse features for outcome at the start of treatment were increasing age $(\mathbf{p}<0.01$ ) and current cigarette smoking (relative risk ( $95 \%$ confidence interval) $2.28(0.93$ to 4.84$)$, $\mathrm{p}<0.05)$. Deaths were mainly from cardiac and vascular causes. The incidence of peritonitis in patients on continuous ambulatory peritoneal dialysis was the same in diabetic patients and controls $(49 \%$ in each group remained free of peritonitis after one year), and the survival of renal allografts was not significantly worse in diabetic patients $(p<0 \cdot 5)$.

Conclusions-Renal replacement treatment may give good results in diabetic patients, although the outlook remains less favourable than for non-diabetic patients because of coexistent, progressive vascular disease, which is more severe in older patients.

\section{Introduction}

The 10 years since this renal unit accepted its first diabetic patients for renal replacement treatment have seen the widespread introduction of continuous ambulatory peritoneal dialysis, increasingly successful renal transplantation, and relaxation of acceptance criteria for diabetic and non-diabetic patients with renal failure. ${ }^{12}$ Diabetic patients form an increasing proportion of the workload of most renal units in the United Kingdom. The percentage of all diabetic patients in the United Kingdom starting renal replacement treatment rose from $1.4 \%$ in $1974^{3}$ to $11.4 \%$ in $1985,{ }^{1}$ and that in this unit has now reached $20 \%$. Despite stringent selection of diabetic patients renal replacement treatment has been associated with poorer survival of diabetic than non-diabetic patients. ${ }^{3-5} \mathrm{We}$ know of no report in the United Kingdom of results of dialysis and transplantation in a less selected group, and we present such an analysis in diabetic and matched non-diabetic patients in the same renal unit.

\section{Patients and methods}

PATIENTS

All patients with end stage renal failure starting dialysis or undergoing renal transplantation at this hospital between 1 January 1979 and 31 December 1988 were considered. Clinical indications to start dialysis were symptoms of uraemia or intractable fluid overload. All patients with diabetes mellitus except two were included; one had renal impairment due to glomerulonephritis and the other impairment due to renal calculi.

\section{CONTROL GROUPS}

Non-diabetic renal replacement treatment group-One non-diabetic patient was selected as a control for each diabetic patient starting renal replacement treatment. The patients were matched for sex, year of starting the treatment, and type of dialysis. Within these constraints the control selected was the one whose date of birth was closest to that of the diabetic patient. This control group was used for all analyses except those of survival of patients and allografts after renal transplantation.

Non-diabetic transplant group-A separate control group was necessary to assess the results of renal transplantation, as the diabetic patients and matched 